\title{
Liposomal Drug Delivery of a Major Component of Coconut Oil for the Treatment of Acne Vulgaris in vitro and in vivo
}

\author{
Dissaya Pornpattananangkul ${ }^{1}$
}

\begin{abstract}
Abundantly found in coconut oil, lauric acid (LA), a 12-carbon atom chain free fatty acid, has shown its potent antimicrobial activity against the acne-causing bacteria, Propionibacterium acnes $(P$. acnes). To facilitate the administration of LA and improve its efficacy, nanoliposomes were used to encapsulate LA. In this study, the antimicrobial activity of LA was compared with other free fatty acids and benzoyl peroxide (BPO). Subsequently, LA was incorporated into nano-sized bilayer lipid vesicle (lauric acid liposome, LipoLA). It was demonstrated that the antimicrobial activity of LA in LipoLA was enhanced at low LA concentration. Interaction study also showed that the liposome aids in the fusion between LA and the bacterial membrane, leading to an effective delivery of LA molecules to the target site. More interestingly, in vivo study indicates that antimicrobial activity of LipoLA is well preserved and higher than BPO. Since LA is a natural compound from coconut oil and liposomes have been successfully and widely applied as a drug delivery vehicle in the clinic, the LipoLA developed in this work holds great potential of becoming a safe and effective therapeutic medication for acne vulgaris and other $P$. acnes associated infections.
\end{abstract}

Keywords: Free fatty acid, coconut oil, lauric acid, acne vulgaris, antimicrobial, drug delivery, liposome, in vivo

\section{Introduction}

Acne vulgaris is a skin disease that is most common during adolescence, afflicting more than $85 \%$ of teenagers and over 40 million people in the United States alone [1,2]. Acne is inflammatory and is associated with the immune response to Propionibacterium acnes ( $P$. acnes), a Gram-positive bacterium that colonizes sebum-rich follicles [3]. The entire genome analysis of $P$. acnes has revealed numerous genes that regulate products involved in degrading host molecules and triggering inflammation [4]. It has been reported that $P$. acnes releases chemoattractants that attract the immune system cells such as neutrophils, monocytes and lymphocytes $[5,6]$. Previous studies have also found that $P$. acnes stimulates the production of pro-inflammatory cytokines such as interleukins-1 $\beta,-8,-12$, and tumor necrosis factor- $\alpha$ [7]. Moreover, inflammatory acne lesions can lead to various degrees of scarring, affecting around 20\% of teenagers and can persist into adulthood. As the disease affects highly visible skin, such as face, chest, and back, it is considered as a critical self-limiting condition. For example, the symptom is mostly developed during adolescence, which is a period that is crucial for building self-esteem and confidence. As a result, there are a number of studies reported about effects of acne on psychological development, including depression, social inhibition, suicidal ideation, and psychosomatic disorders.

\footnotetext{
${ }^{1}$ Department of Chemistry, Faculty of Science, Chulalongkorn University, Pathumwan, Bangkok, 10330 Thailand.

Email: nudissaya@gmail.com
} 
To solve problems of OTC drugs, we have thoroughly studied the anti-acne property of lauric acid, a major component in coconut oil. Lauric acid is the majority component of coconut oil, making up around 50\%. This report aims to summarize all aspects of LA related to its antiacne activity, starting from its antimicrobial activity comparing to other FFAs and thoroughly study of an encapsulation of this molecule into nanoparticles both in vitro and in vivo $[8,9,10]$.

In this study, we selected liposomes as a drug carrier. Liposomes are lipid bilayer vesicle which are one of the most extensively explored drug carriers since early 1980s with high therapeutic effectiveness [11, 12]. Liposomes can enhance dermal and transdermal drug delivery while reducing systemic absorption because of the similarity in lipid composition to the epidermis [13]. The study on liposomes for targeting drugs into the pilosebaceous units has suggested that liposomes are potent drug delivery systems for treating hair follicleassociated disorders such as acne [11]. In fact, Lieb et al. has proved that liposomes delivered much higher drug concentrations to the pilosebaceous unit than conventional drug formulations [14]. During the past few decades, liposomes have been used as carriers to enhance clinical efficacy for a large number of drugs. Pevaryl Lipogel as the first topical liposomal drug in the market was launched in 1988 [11]. In addition, antiacne drug-loaded liposomes such as tretinoin, clindamycin, salicylic acid, and tea tree oil-loaded liposomes have been recently reported [15 - 17].

Here we use liposomes to encapsulate LA and deliver it to $P$. acnes. LA is an amphiphilic molecule consisting of a hydrophobic hydrocarbon chain and a hydrophilic carboxylic acid headgroup. (Note: Is it in the neutral carboxylic acid form or the anionic carboxylate form? Is there evidence that indicates which form is present? Naturally, LA is a component of triglycerides, and it does not present in an anionic form. Ref: N.H. Jayadas et al., Tribology International, 2006. 39: p. 873-878.: Its amphiphilic nature makes it a good candidate to be incorporated into the bilayered wall of liposomes that provides an amphiphilic environment. In this study, complete investigation of LA-loaded liposome from characterization, interaction mechanism, and antimicrobial activity both in vitro and in vivo is reported.

\section{Materials and methods}

\subsection{Materials}

Hydrogenated L-a-Phosphatidylcholine (Egg PC), cholesterol, C6-NBD Phytosphing, and 1, 2-Dimyristoyl-sn-Glycero-3Phosphoethanolamine-N-Lissamine Rhodamine B Sulfonyl (DMPE-RhB) were purchased from Avanti Polar Lipids, Inc. (Alabaster, AL). Lauric acid (LA) and 18-crown-6 were obtained from Sigma Aldrich (St Louis, MO). $\mathrm{KHCO}_{3}$ was from Fisher Scientific (Pittsburgh, PA). 3, 4difluorophenacyl bromide was purchased from Maybridge (Cambridge, UK). Brucella broth, Gas-Pak, and agar were purchased from BD (Sparks, MD). Reinforced clostridium medium was from Oxoid (Hampshire, UK). In order to prepare carboxyl functionalized gold nanoparticles (AuC), the following chemicals were purchased: hydrogen tetrachloroaurate $\left(\mathrm{HAuCl}_{4}\right)$ (ACROS Organics), Sodium borohydride $\left(\mathrm{NaBH}_{4}\right)$ (ACROS Organics), and 3-Mercaptopropionic acid (MPA) (Sigma Aldrich).

\subsection{Preparation and characterization of LA-loaded liposomes (LipoLA)}

Large unilamellar LA-loaded liposomes (LipoLA) were prepared by the well-known vesicle extrusion technique [16]. Briefly, liposomes composed of $4.5 \mathrm{mg}$ of Egg PC, cholesterol and LA (9:1:0, 8.5:1:0.5, 8:1:1, 7:1:2 and $5: 1: 4$, weight ratio, respectively) were dissolved in $3 \mathrm{~mL}$ of chloroform, which was then evaporated by leading argon gas for 15 minutes. The resultant lipid films were then stored over night under high vacuum to remove any traces of chloroform. The dried lipid films were then rehydrated with $3 \mathrm{~mL}$ of deionized water or sterile PBS buffer $(1 \mathrm{X}, \mathrm{pH}=7.4)$. The suspensions of lipid were vortexed for 5 minutes and then sonicated for 10 minutes in a bath sonicator (Fisher Scientific FS30D) to produce multilamellar vesicles (MLVs). A Ti-probe 
(Branson 450 sonifier) was used to sonicate the MLVs for 1-2 minutes at $20 \mathrm{~W}$ to produce small unilamellar vesicles (SUVs). The resulting SUVs were then extruded through a $100 \mathrm{~nm}$ pore-sized polycarbonate membrane for 11 times to form the final products of LipoLA.

\subsection{Antimicrobial activity of LipoLA}

To determine the antimicrobial activity of LipoLA, two sets or samples were investigated.

(i) LipoLA with a constant LipoLA concentration and a LA loading concentration of $0,12,33,80$, and $102 \mu \mathrm{g} / \mathrm{mL}$, respectively. In this set, the LipoLA concentration of each sample was constant while the LA concentration per liposome was different. (ii) LipoLA with a LA loading concentration of 25.5, 51, and 102 $\mu \mathrm{g} / \mathrm{mL}$, respectively. However, the $25.5 \mu \mathrm{g} / \mathrm{mL}$ LipoLA and the $51 \mu \mathrm{g} / \mathrm{mL}$ LipoLA were obtained by diluting the $102 \mu \mathrm{g} / \mathrm{mL}$ LipoLA solution with PBS fourfold and twofold, respectively. In this set, the LipoLA concentration of each sample was different while the LA concentration per liposome was constant. PBS (1X, pH=7.4) and empty liposomes (without LA) were used as negative controls. All samples were incubated with $P$. acnes $\left(1 \times 10^{7}\right.$ $\mathrm{CFU} / \mathrm{mL}$ ) at $37^{\circ} \mathrm{C}$ for 5 hours under anaerobic condition. After incubation, the samples were diluted $1: 10$ to $1: 10^{6}$ in PBS, and $5 \mu \mathrm{L}$ of dilutions was spotted on Brucella Broth agar plates supplemented with 5\% defibrinated sheep blood and hemin and vitamin K. Agar plates were incubated at $37^{\circ} \mathrm{C}$ under anaerobic condition for 3 days, and CFU (colony forming units) of $P$. acnes was quantified.

\subsection{Bacteria fusion studies}

Förster resonance energy transfer (FRET) was preformed to investigate the interaction mechanism between LipoLA and P. acnes. To prepare a FRET-pair labeled LipoLA, a florescent donor $\left(\mathrm{C}_{6} \mathrm{NBD}, 0.1 \mathrm{~mol} \%\right)$ and a fluorescent quencher (DMPE-RhB, $0.5 \mathrm{~mol} \%$ ) were simultaneously incorporated into the LipoLA (102 $\mu \mathrm{g} / \mathrm{mL} \mathrm{LA)} \mathrm{by} \mathrm{mixing} \mathrm{the} \mathrm{donor}$ and acceptor with eggPC, cholesterol, and LA before the formation of LipoLA. The resulting LipoLA was then diluted with PBS twofold to prepare LipoLA $(51 \mu \mathrm{g} / \mathrm{mL}$ LA). Subsequently, the diluted LipoLA solutions were mixed with $P$. acnes $\left(8 \times 10^{7}, 1.2 \times 10^{8}, 1.6 \times 10^{8}\right.$, and $3.2 \times 10^{8}$ $\mathrm{CFU} / \mathrm{mL}$, respectively). The total volume of the final solution (LipoLA $+P$. acnes) was $1 \mathrm{~mL}$. After $10 \mathrm{~min}$ incubation at room temperature, samples were centrifuged at $13,500 \mathrm{rpm}$ for 1 min to remove the excess amount of LipoLA and were resuspended in PBS $(1 \mathrm{~mL})$. Consequently, emission spectra in the region of $500-700 \mathrm{~nm}$ were obtained by exciting the sample at $470 \mathrm{~nm}$ using fluorescent spectrophotometer (Infinite M200, TECAN, Switzerland). Solution of LipoLA $(51 \mu \mathrm{g} / \mathrm{mL}$ LA) without incubating with $P$. acnes was used as a negative control. Fluorescence emission of each sample was subtracted with background signal, which was the emission of $P$. acnes itself with the corresponding concentration.

\subsection{Antimicrobial activity of LipoLA in vivo-intradermal injection model}

Effect of LipoLA on $P$. acnes was determined in a physiological environment using ICR mouse ears. Right before injection, LipoLA $(8 \mathrm{mg} / \mathrm{mL})$ was mixed with $P$. acnes $\left(10^{8}\right.$ $\mathrm{CFU} / \mathrm{mL})$. The resulting solution $(10 \mu \mathrm{L})$ was intradermally injected into the ear of mice. P. acnes in benzoyl peroxide $(16 \mathrm{mg} / \mathrm{mL})$ in $5 \%$ DMSO and PBS served as a positive and negative control respectively. The ears were collected $24 \mathrm{hrs}$ after injection, using a $8 \mathrm{~mm}$ biopsy punch and homogenized in $1 \mathrm{~mL}$ of sterile PBS with homogenizer (MiniBeadbeater $\left.{ }^{\mathrm{TM}}\right)$. Homogenates were diluted 1:10 to $1: 10^{6}$ in PBS, and $10 \mu \mathrm{L}$ of each dilution was spotted on RCM agar plates. Then, the agar plates were incubated at $37^{\circ} \mathrm{C}$ under anaerobic condition for 3 days, and the CFU (colony forming units) of $P$. acnes was quantified. Ears without $P$. acnes inoculation served as a negative control to ensure that there was no contamination from other bacteria. The study was performed with six separate experiments.

\section{Results and discussions}

Firstly, antimicrobial of lauric acid was compared with other free fatty acids, including palmitic acid (PA) and oleic acid (OA) to determine its specificity against $P$. acnes. It was found that at the concentration of $<100 \mu \mathrm{g} / \mathrm{mL}$, 
PA and OA did not show any antimicrobial activity, while LA completely killed bacteria at $80 \mu \mathrm{g} / \mathrm{mL}$. This result indicates that among three different types of free fatty acids from coconut oil, LA showed the highest antimicrobial activity against $P$. acnes and there is specificity against this bacteria [Figure 1]. Only LA has significant activity against the bacteria, while PA and OA do not. This indicates that there is specificity of specific type of free fatty acid against the bacteria. More interestingly, incubation of $P$. acnes with lauric acid yielded minimal inhibitory concentration (MIC) values against the bacterial growth over 15 times lower than those of benzoyl peroxide (BPO). The lower MIC values of lauric acid indicate stronger antimicrobial properties than that of BPO [Table 1]. To enhance its activity and improve drug delivery property to the targeted site, a nanoparticle of this molecule was developed. Lauric acid-loaded liposomes (LipoLA) with a diameter of $\sim 100 \mathrm{~nm}$ were prepared. The liposome around this size was selected for this study due to the fact that smaller liposomes with diameters near $100 \mathrm{~nm}$ have shown improved penetration through skin comparing to larger ones [12]. As shown in Figure 2, the z-average mean size of LipoLA was in the range of $113.6 \pm 1.6 \mathrm{~nm}$ and $123.6 \pm$ $1.5 \mathrm{~nm}$ when the initial LA input varied from $0 \sim 200 \mu \mathrm{g} / \mathrm{mL}$. These results suggest that the increase of LA concentration has no significant effect on the liposome size. Moreover, the zetapotentials of LipoLA formulated with $0,25,50$, 100 , and $200 \mu \mathrm{g} / \mathrm{mL}$ initial LA input were $-8.4 \pm$ $0.1,-23.3 \pm 0.9,-29.3 \pm 1.2,-37.7 \pm 0.4$ and $51.1 \pm 3.3 \mathrm{mV}$, respectively. Free fatty-acids have a $\mathrm{pK}$ value of approximately 5, thus at near physiological $\mathrm{pH}$ of 7.4 the carboxyl group of the free fatty acid will deprotonate and attribute to the negatively charged surface of the liposome. Liposomes are lipid vesicles that are prone to fuse among themselves. To prevent an attachment and fusion between each liposome, slightly negatively charged liposomes with zeta potential around $-30-50 \mathrm{mV}$ have to be formulated. In this case, liposomes cannot fuse among themselves and their shelf life will be prolonged. This explains the observation of an increase of LA concentration in liposome leading to a decrease of the zeta-potential, and

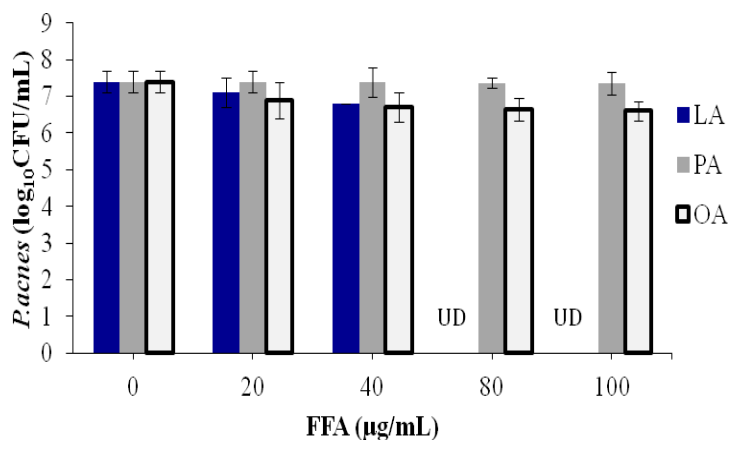

Figure 1. Antimicrobial effects of free fatty acids against $P$. acnes. $P$. acnes $\left(1 \times 10^{7}\right.$ CFU $/ \mathrm{mL}$ ) was incubated with $0-100 \mu \mathrm{g} / \mathrm{mL}$ of lauric acid (LA), palmitic acid (PA), or oleic acid (OA) in 5\% DMSO in PBS for 5 hours under anaerobic conditions and amount of $P$. acnes was quantified. Data represent mean $\pm \mathrm{SE}$ of three individual experiments $(* * P<0.005$ by Student's $t$-test).

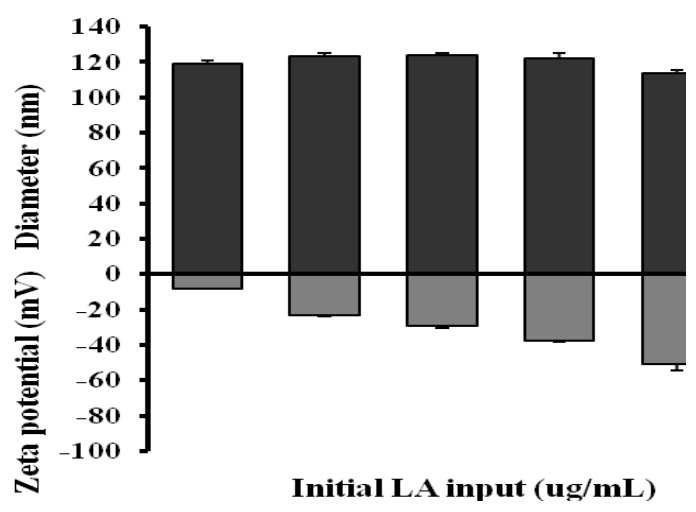

Figure 2. Characterization of lauric acid-loaded liposomes (LipoLA). LA at various concentrations ranging from $0 \sim 200 \mu \mathrm{g} / \mathrm{mL}$ was mixed with other lipid components to prepare LipoLA. The size (diameter, nm) and surface zeta potential $(\mathrm{mV})$ of the LipoLA were determined by dynamic light scattering.

Table 1. MIC and EC50 of lauric acid and BPO against $P$. acnes

\begin{tabular}{|c|c|c|}
\hline & $\mathrm{MIC}(\mu \mathrm{g} / \mathrm{mL})$ & $\mathrm{EC}_{50}(\mu \mathrm{g} / \mathrm{mL})$ \\
\hline Lauric Acid & 3.9 & 2 \\
\hline BPO & 62.5 & 60 \\
\hline
\end{tabular}


confirms that LA is indeed incorporated into the liposomes. Using HPLC, drug encapsulation efficiency of LA in LipoLA (initial LA input of $200 \mu \mathrm{g} / \mathrm{mL}$ ) is $51 \%$. The high yield results from an encapsulation of LA in the hydrophobic membrane, not from the hydrophilic core. Typically, loading yield of hydrophilic drug in liposome for drug delivery is low (Ref: Anthony D. Duong et al., One Step Encapsulation of Small Molecule Drug in Liposome Via Electrospray-Remote Loading. Mol. Pharmaceutics, 2015) because during preparation process, encapsulated hydrophilic drug inside an aqueous core cannot enclosed high drug concentration and not many drug molecules can be encapsulated inside. In this case, however, the drug concentration is high because LA is lipophilic and is a component of the lipid membrane itself, so it is a major component of the lipid layer that is formed; thus, this results in high loading yield.

To study antimicrobial effect of LipoLA, Five samples of LipoLA $(0,12,33,80$, and 102 $\mu \mathrm{g} / \mathrm{mL}$ LA) were incubated with $P$. acnes $\left(1 \times 10^{7}\right.$ $\mathrm{CFU} / \mathrm{mL}$ ) for 5 hours under anaerobic condition in order to determine their antimicrobial activity. The result showed that LipoLA with $102 \mu \mathrm{g} / \mathrm{mL}$ LA completely killed the bacteria (Figure 3A). In these five samples, the LA loading concentration was different but the molar concentration of liposome in each sample was kept constant. To further investigate the system, LipoLA with $102 \mu \mathrm{g} / \mathrm{mL}$ LA was diluted twofold and fourfold to obtain 51 and 25.5 $\mu \mathrm{g} / \mathrm{mL}$ LipoLA, respectively, and their antimicrobial activities were determined. In this experiment, the results showed that $P$. acnes was completely killed by $51 \mu \mathrm{g} / \mathrm{mL}$ LipoLA (Figure $3 \mathrm{~B})$. This observation indicated that the higher molar ratio of LA in each liposome is a critical factor for drug efficacy because the interaction space on the bacteria is limited. When liposomes interact with bacteria, they bind onto bacterial cell. The interaction space here refers to space on bacterial cell wall. Bacteria has specific size and specific area of membrane around the cell. Thus, the area, or interaction space, that liposomes can bind to is limited. Higher loading in one liposomes results in effective treatment because liposome number that can bind to bacteria is limited. Moreover, the antimicrobial activity of LA in the liposomes was more efficient than free molecules.
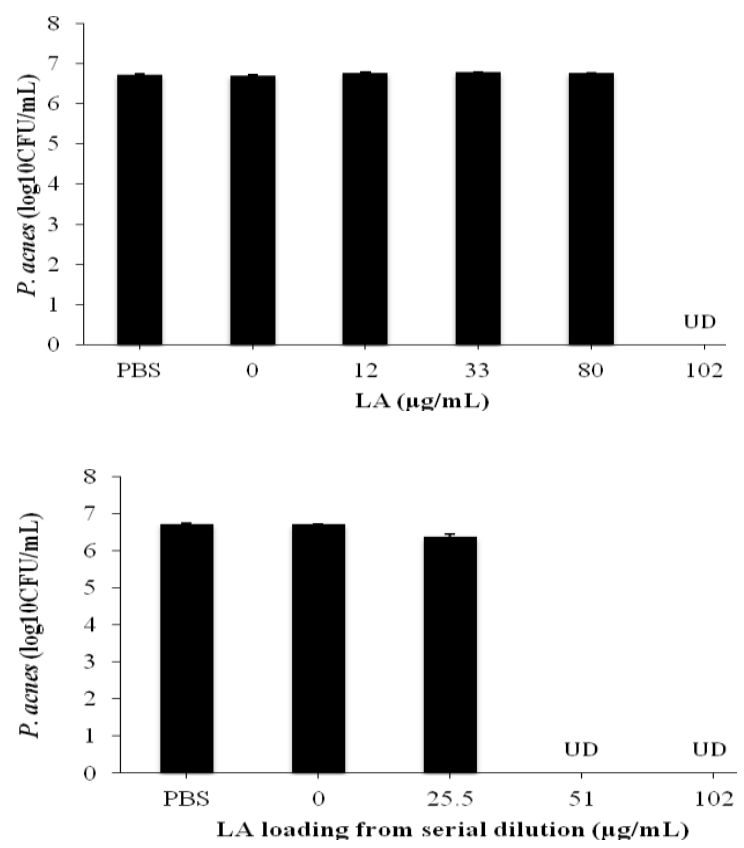

Figure 3. Antimicrobial activity of LipoLA against $P$. acnes. Two sets of LipoLA were incubated with $P$. acnes $\left(1 \times 10^{7} \mathrm{CFU} / \mathrm{mL}\right)$, respectively, for 5 hours under anaerobic conditions to test their antimicrobial activity. (A) LipoLA with a LA loading concentration of 0 , $12,33,80$, and $102 \mu \mathrm{g} / \mathrm{mL}$, respectively. In this set, the LipoLA concentration of each sample was constant while the LA concentration per liposome was different. The results showed that $102 \mu \mathrm{g} / \mathrm{mL}$ LipoLA completely killed $P$. acnes. (B) LipoLA with a LA loading concentration of $25.5,51$, and $102 \mu \mathrm{g} / \mathrm{mL}$, respectively. However, the $25.5 \mu \mathrm{g} / \mathrm{mL}$ LipoLA and the $51 \mu \mathrm{g} / \mathrm{mL}$ LipoLA were obtained by diluting the 102 $\mu \mathrm{g} / \mathrm{mL}$ LipoLA solution with PBS fourfold and twofold, respectively. In this set, the LipoLA concentration of each sample was different while the LA concentration per liposome was constant. The results showed that both $51 \mu \mathrm{g} / \mathrm{mL}$ LipoLA and $102 \mu \mathrm{g} / \mathrm{mL}$ LipoLA completely killed bacteria. Incubation with PBS buffer and empty liposome solution (without LA) served as negative controls. Data represent mean \pm SD of three individual experiments. UD: undetectable. 


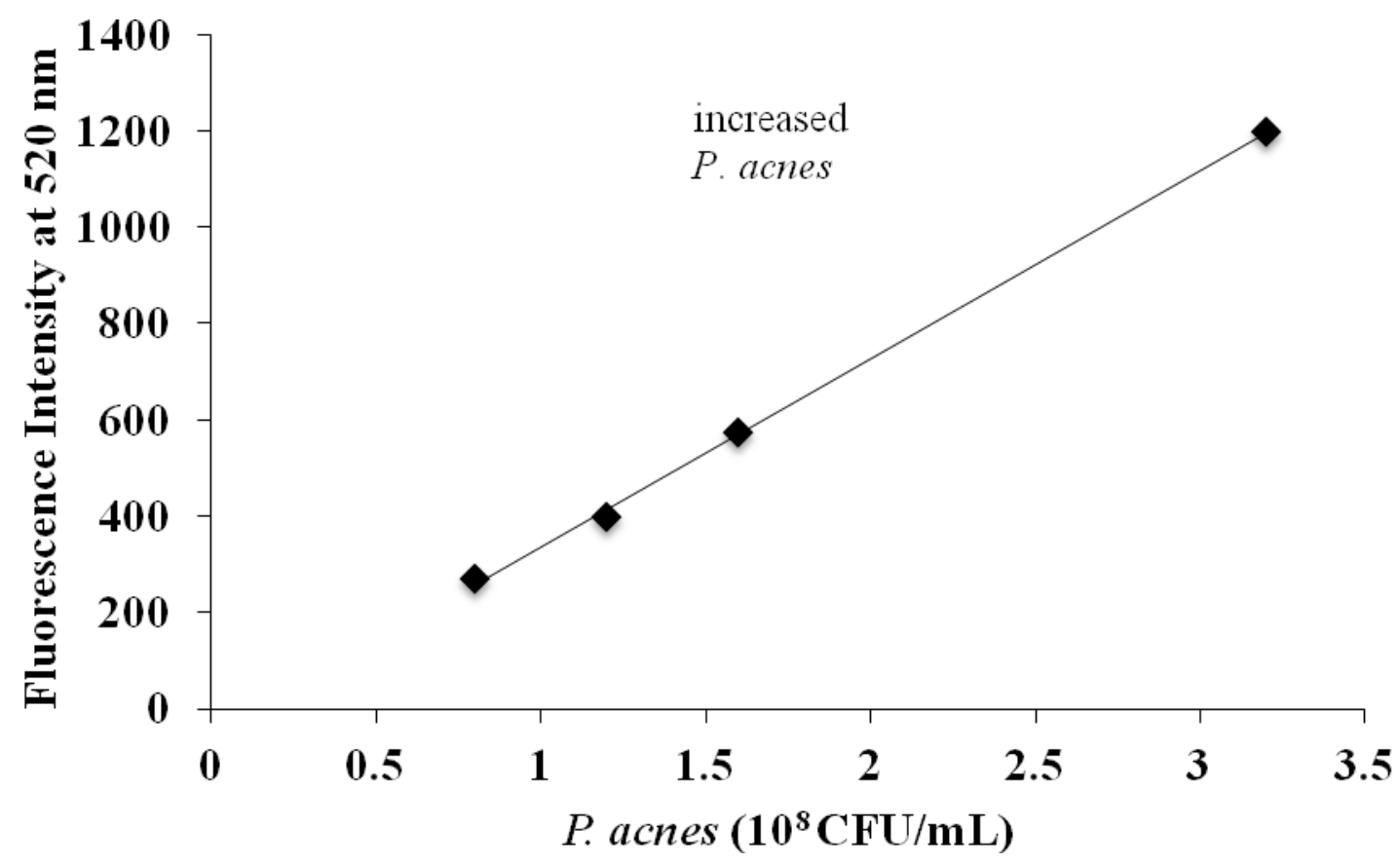

Figure 4. FRET measurements of the fusion between LipoLA and P. acnes. A fluorescent donor $\left(\mathrm{C}_{6} \mathrm{NBD}\right)$ and a fluorescent quencher (DMPE-RhB) were simultaneously incorporated into the LipoLA $(51 \mu \mathrm{g} / \mathrm{mL}$ LA) with a proper molar ratio that the quencher effectively quenched the fluorescence emission from the donor. The FRET-pair labeled LipoLA was incubated with $P$. acnes at a concentration of $8 \times 10^{7}, 1.2 \times 10^{8}$, $1.6 \times 10^{8}$, and $3.2 \times 10^{8} \mathrm{CFU} / \mathrm{mL}$ for 10 minutes. After removing the excess LipoLA, all samples were excited at $470 \mathrm{~nm}$ and fluorescence emission was recorded (inset). A rise in emission intensity of $\mathrm{C}_{6} \mathrm{NBD}$ (donor) at $520 \mathrm{~nm}$ was observed with the increasing bacterial concentration, indicating the occurrence of fusion between LipoLA and bacteria that caused the spatial separation of $\mathrm{C}_{6} \mathrm{NBD}$ and DMPE-RhB.

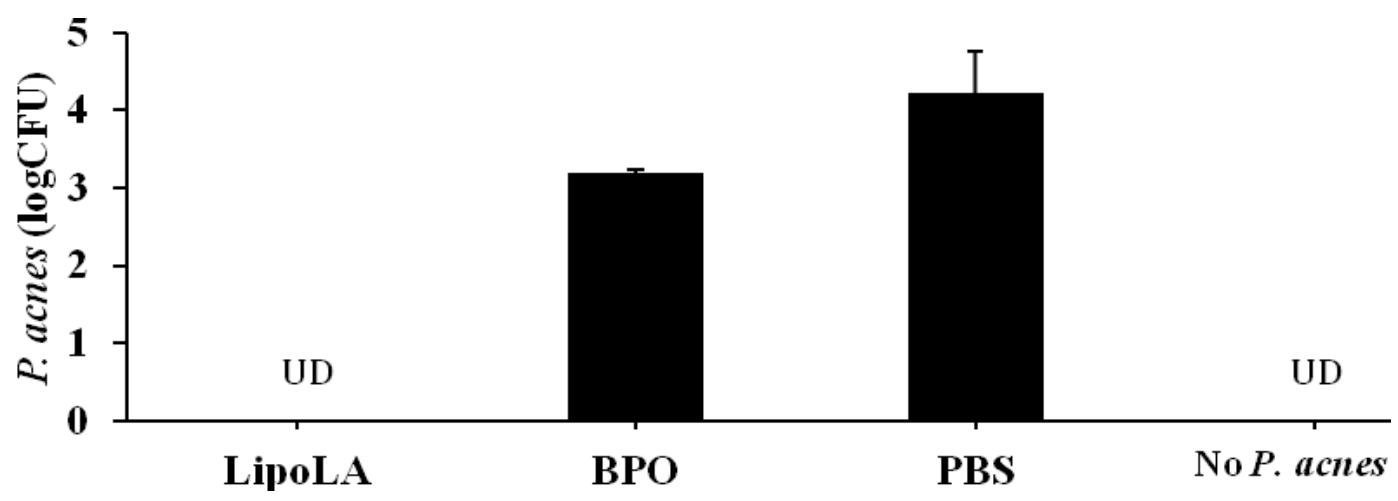

Figure 5. In vivo antimicrobial activity of LipoLA studied by an intradermal injection model. LipoLA (8 $\mathrm{mg} / \mathrm{mL}$ ) was mixed with $P$. acnes and immediately injected into mouse ears, following with bacteria quantification at 24 hours. BPO $(16 \mathrm{mg} / \mathrm{mL})$ was a positive control. PBS and none P. acnes inoculation were served as negative controls. Data represents six individual experiments. UD $=$ undetectable. 
In order to understand the interaction mechanism between LipoLA and the bacteria, FRET assay was performed. FRET is a widely used technique that precisely measures the distance of two subjects at the molecular level based on an energy transfer mechanism of two chromophores [18]. During the fusion between LipoLA and bacterial membranes, the spread of the donor $\left(\mathrm{C}_{6} \mathrm{NBD}\right.$ : excitation/emission $=470$ $\mathrm{nm} / 520 \mathrm{~nm}$ ) and acceptor (DMPE-RhB: excitation/emission $=550 \mathrm{~nm} / 580 \mathrm{~nm}$ ) within the bacterial membranes will eliminate the FRET efficiency, resulting in fluorescence recovery of the donor. As shown in Figure 4, the rise in the emission peak of $\mathrm{C}_{6} \mathrm{NBD}$ at $520 \mathrm{~nm}$ was detected when the concentration of $P$. acnes increased indicating spatial separation between $\mathrm{C}_{6} \mathrm{NBD}$ and DMPE-RhB increased and the efficiency of energy transfer decreased. LipoLA at the same concentration while not mixing with bacteria was used as a control for this study. When the control sample was excited at $470 \mathrm{~nm}$, no emission at $520 \mathrm{~nm}$ was detected but the emission peak at $580 \mathrm{~nm}$ was observed. The changes of the emission peak of DMPE-RhB was neglected in this study because DMPE-RhB could be excited by not only the FRET from the donor, but also by excitation at $470 \mathrm{~nm}$. These observations indicate that the interaction mechanism between LipoLA and P. acnes was fusion; not adsorption or aggregation. As shown in Figure 4, changes in donor emission were observed indicating insertion of liposomal membrane between donor and acceptor chromophores. In case of adsorption or aggregation, however, there will not be any insertion of bacterial membrane to liposomes. There will only be an attachment between two vesicles, and no changes in FRET signals between donors and acceptors will be observed.

This fusion mechanism is also consistent with previous reports of liposomal drug delivery to gram-positive bacteria such as Staphylococcus aureus and Stenotrophomonas maltophilia [19].

The efficacy of our liposomal formulation in vivo by intradermal injection into ICR mouse ear was further observed. The ear was selected for this study because of its confined structure in order to ensure that all of the inoculated bacteria will remain inside of the injection area. As shown in Figure 5, comparing to the bacteria number of the untreated sample of 4.22 $\log (\mathrm{CFU} / \mathrm{mL})$, LipoLA can completely kill $P$. acnes in this condition. Considering to the positive control, $16 \mathrm{mg} / \mathrm{mL}$ of $\mathrm{BPO}$ in $5 \%$ DMSO, there was a reduction in number of bacteria from 4.22 to 3.20 , which was only about one order of magnitude reduction. BPO was selected as a positive control because it is a widely used OTC acne treatment. It is commonly prepared in many dosage forms, including cream, gel, or lotion, all of which are easy and convenient to apply, but are not suitable for intradermal injection. Thus, in this study, a solution of BPO was prepared in 5\% DMSO, which is the condition that does not generate acute toxicity to human sebocytes or $P$. acnes [20].

\section{Conclusions}

In conclusion, lauric acid (LA), a major component in coconut oil, indicated highest antimicrobial activity against Propionibacterium acnes ( $P$. acnes), the major causing agent of acne vulgaris. We developed lauric acid-loaded liposomes (LipoLA) to effectively deliver this molecule to the targeted site. According to LipoLA characterization, the size of LipoLA in the range of $110-120 \mathrm{~nm}$ with negative charge not exceed $-50 \mathrm{mV}$ indicates both a suitable size distribution for skin delivery as well as the full encapsulation of lauric acid (LA), a negatively charged antimicrobial free fatty acid within the liposome. It was demonstrated that its antimicrobial activity against $P$. acnes was well maintained and even enhanced at low LA concentration. This study also showed that the antimicrobial activity of LipoLA mainly depended on the LA loading concentration per single liposomes. Further study disclosed that LipoLA could fuse with the membranes of $P$. acnes and release the carried LA directly into the bacterial membranes. More interestingly, the in vivo study indicates that the antimicrobial activity is well preserved. Since liposomes have been widely used in cosmetics products and also approved for use in the clinic as a drug delivery vehicle, and LA is the natural component from coconut oil, the LipoLA developed in this work 
has the potential of becoming an innate, safe and effective therapeutic medication for acne vulgaris and other $P$. acnes associated diseases.

\section{Acknowledgements}

The author acknowledges a financial support from Rachadapisek Sompot Fund for Postdoctoral Fellowship, Chulalongkorn University and would love to thank Prof. Supasorn Wanichwecharungruang, Prof. Sirirat Rengpipat, Ratthakan Deephum, and Pawatsanai Samutprasert regarding to in vitro antimicrobial study at Chulalongkorn University, Bangkok, Thailand. Moreover, the author thanks to Zhang's Research Group at University of California, San Diego for collaboration of every experiments in this work.

\section{References}

Beaulac, C., S. Sachetelli, and J. Lagace, In-vitro bactericidal efficacy of sub-MIC concentrations of liposome-encapsulated antibiotic against gram-negative and gram-positive bacteria. J Antimicrob Chemother, 1998. 41(1): p. 35-41.

Biju, S.S., A. Ahuja, and R.K. Khar, Tea tree oil concentration in follicular casts after topical delivery: determination by highperformance thin layer chromatography using a perfused bovine udder model. J Pharm Sci, 2005. 94(2): p. 240-5.

Bhalerao, S.S. and A. Raje Harshal, Preparation, optimization, characterization, and stability studies of salicylic acid liposomes. Drug Dev Ind Pharm, 2003. 29(4): p. 451-67.

Bojar, R.A. and K.T. Holland, Acne and Propionibacterium acnes. Clin Dermatol, 2004. 22(5): p. 375-9.

Bruggemann, H., et al., The complete genome sequence of Propionibacterium acnes, a commensal of human skin. Science, 2004. 305(5684): p. 671-3.

Burkhart, C.G., C.N. Burkhart, and P.F. Lehmann, Acne: a review of immunologic and microbiologic factors. Postgrad Med J, 1999. 75(884): p. 328-31.
Fried, R.G. and A. Wechsler, Psychological problems in the acne patient. Dermatol Ther, 2006. 19(4): p. 237-40.

Ha, T., Single-molecule fluorescence resonance energy transfer. Methods, 2001. 25(1): p. 78-86.

Honzak, L. and M. Sentjurc, Development of liposome encapsulated clindamycin for treatment of acne vulgaris. Pflugers Arch, 2000. 440(5 Suppl): p. R44-5.

Kim, J., Review of the innate immune response in acne vulgaris: activation of Toll-like receptor 2 in acne triggers inflammatory cytokine responses. Dermatology, 2005. 211(3): p. 193-8.

Lieb, L.M., et al., Topical delivery enhancement with multilamellar liposomes into pilosebaceous units: I. In vitro evaluation using fluorescent techniques with the hamster ear model. J Invest Dermatol, 1992. 99(1): p. 108-13.

Mezei, M. and V. Gulasekharam, Liposomes-a selective drug delivery system for the topical route of administration: gel dosage form. J Pharm Pharmacol, 1982. 34(7): p. 473-4.

Nakatsuji, T., et al., Antimicrobial property of lauric acid against propionibacterium acnes: Its Therapeutic Potential for Inflammatory Acne Vulgaris. $\mathrm{J}$ Invest Dermatol, 2009. 129(10): p. 2480-8.

Nakatsuji, T., et al., Sebum free fatty acids enhance the innate immune defense of human sebocytes by upregulating betadefensin-2 expression. J Invest Dermatol. 130(4): p. 985-94.

Pornpattananangkul, D., et al., In vivo treatment of Propionibacterium acnes infection with liposomal lauric acids. Adv Healthcare Mater, 2013. 2(10): p. 1322-1328.

Schmid, M.H. and H.C. Korting, Therapeutic progress with topical liposome drugs for skin disease. Adv Drug Delivery Rev, 1996. 18(3): p. 335-342. 
Taglietti, M., C.N. Hawkins, and J. Rao, Novel topical drug delivery systems and their potential use in acne vulgaris. Skin Therapy Lett, 2008. 13(5): p. 6-8.

Verma, D.D., et al., Particle size of liposomes influences dermal delivery of substances into skin. Int J Pharm, 2003. 258(1-2): p. 141-51.

Webster, G.F. and J.J. Leyden, Characterization of serum-independent polymorphonuclear leukocyte chemotactic factors produced by Propionibacterium acnes. Inflammation, 1980. 4(3): p. 261-9.

Yang, D and Pornpattananangkul, D., et al., The antimicrobial activity of liposomal lauric acids against Propionibacterium acnes. Biomaterials, 2009. 30(30): p. 6035-6040. 